\title{
CERVICAL SPONDYLOSIS
}

\author{
By L. A. LiverSedge, M.D., M.R.C.P. \\ Lecturer in Neurology, University of Manchester
}

In the years $195^{6}, 1957$ and $195^{8}$ the diagnostic index of new cases seen in the department of neurology at Manchester Royal Infirmary contained rather more than 140 cases of cervical spondylosis. In the corresponding three years 1946, 1947 and 1948 this diagnosis appears not at all, though one notes a number of cases of osteoarthritis of the cervical spine, a rather smaller number of cases characterized as prolapsed cervical intervertebral disc, and sundry other groups under titles variously described as 'costoclavicular compression,' ' dropped shoulder girdle syndrome' and ' cervical radiculopathy.' These facts contain in themselves no primary statistical significance, but they are important in indicating the remarkable change which has taken place in neurological diagnostic nosology in the last 15 years.

A second point of importance and one which is the direct result of this relatively sudden emergence of a new clinical entity, lies in the inevitable confusion about the aetiology of the bony and cartilaginous disturbance in the cervical spine and its resultant neurological defects. The term ' cervical spondylosis' as used by differing authorities has tended to embrace a number of different processes comprising degeneration of the vertebrae and discs, osteoarthritic changes at the intervertebral joints, disc herniation and osteophyte formation. In many cases these changes occur simultaneously but the terms have tended to be used with a certain lack of discrimination mainly as a result of the short period of observation which is so far available for the correct study of these disorders. As time has gone by, observers, largely stimulated by the careful and thoughtful work of Brain, 1, 2 have been at great pains to describe in detail the extraordinarily varied clinical picture which may result from pathological changes in the cervical spine, its intervertebral discs, and the associated intervertebral foramina. There has now emerged a composite picture of the effects of this bony and cartilaginous disorganization upon the spinal cord and the adjacent nerve roots, and it will be abundantly clear that the widely varying pictures simulating such classical neurological conditions as motor neurone disease, syringomyelia, spinal disseminated sclerosis and progressive lateral sclerosis, will depend upon the way in which the bony and cartilaginous tissues press upon the cord, the roots, or even upon the vessels responsible for the normal functioning of these structures. To take a single and commonly occurring neurological example, the degeneration of an intervertebral disc in the cervical region is usually followed by the production of osteophytes on the posterior aspects of the adjacent vertebrae. This reactive osteophyte formation results, in turn, in pressure on the anterior aspect of the spinal cord and probably at the same time upon the anterior spinal artery. In this way it produces a steadily progressive ischaemia, probably aggravated by the natural movements of the neck and in many cases conditioned by the room available in the spinal neuralo canal. Payne and Spillane ${ }^{3}$ have drawn attention to the importance of the antero-posterior diametere of the spinal canal, and have shown that it is in the narrower type of canal that spinal myelopathy tends to be produced. In certain patients with an advanced degree of radiological change in the cervical spine no neurological disorder may be detected, and no symptoms may ever develop. The more lateral production of osteophytes often associated with a tear of the annulus of the disc, may result in a compression of the antero-lateral spinal thalamic tract, and so give a picture which may resemble syringomyelia. If the site of compression is still more lateral the stress falls upon the anterior roots and since more than one root may be involved, particularly in a spine which is affected fairly diffusely, then the presenting signs and symptoms may be almost indistinguishable from progressive muscular atrophy. Our present concern, however, is not with the widely varying neurological pictures of myelopathy but with the problem of pain in the arm and its relation to affections of the cervical spine. In considering this particular problem, it is important in the first instance to clarify briefly the pathological changes which may currently be included in the term ' cervical spondylosis.'

\section{Acute Prolapse of a Cervical Disc}

It is certainly true that in the early stages our views on cervical spondylosis were very largely 
conditioned by the pre-existing views on prolapsed lumbar intervertebral disc, and for many people the two terms were largely synonymous. The notion was entertained, whether overtly or not, that flexion and extension of the cervical spine might produce prolapse of the cervical intervertebral disc, in much the same way as an acute prolapse took place in the lumbar region. We are indebted to Frykholm ${ }^{4}$ for pointing out to us the relative infrequency of soft nuclear herniations in the cervical region, analagous to those seen so frequently in the lumbar area. Nevertheless, from time to time one does encounter, in relatively young people, symptoms which can only be regarded as attributable to a sudden lateral herniation of a cervical intervertebral disc with acute pain down the arm, relieved fairly rapidly by a period of immrtilization and rest.

\section{The Generalized Process of Osteoarthritic Wear and Tear}

This may include any part of the spine, but is more commonly seen in the lumbar region. In many cases, however, the cervical vertebrae and their associated joints share in the osteoarthritic process. Such cases may present either with relatively ill-localized pain in the neck, often aggravated by movement, and in certain circumstances the picture may be very much of radicular type as a result of osteophytic narrowing of the intervertebral foramina, with consequent pressure upon the nerve roots as they pass outwards from the dural canal. This is scarcely the place in which to join in the mild controversy set up about the existence or otherwise of the neurocentral joints of Lushka. Suffice to say that although Cave et al. ${ }^{5}$ stressed the existence of a separate neurocentral joint and commented upon the frequency of Lushka joint arthritis in cases in which neurological syndromes had developed, the more recent work of Payne and Spillane ${ }^{3}$ denies categorically the existence of these particular joints and re-emphasizes Frykholm's conclusion that it was not justifiable to speak of these as being separate uncovertebral or neurocentral joints. From our point of view, this pathological discussion is perhaps of no great clinical significance, but one feels that there is some ground for the contention that osteoarthritic changes in the lateral portion of the adjacent vertebral surfaces may, in fact, result in narrowing of the intervertebral foramina, and so produce the pain in the arm with which we are now clinically concerned.

\section{Cervical Spondylosis}

The term 'spondylosis' implies a degenerative process of aetiology not necessarily established.
As Payne and Spillane ${ }^{3}$ have pointed out the process of ageing appears to result in sclerotic $\varrho$ changes in the cervical vertebrae and since the $\frac{\mathbb{\Phi}}{\mathbb{Q}}$ nutrition of the avascular intervertebral disc is by $\stackrel{\varrho}{c}$

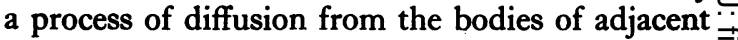
vertebrae through the cartilaginous end plates, it $\stackrel{\bar{s}}{3}$ may well be that the process we know as ' disco degeneration' may result from a change which 흐 actually begins within the vertebral bodies or in $\overline{\frac{\bar{T}}{T}}$

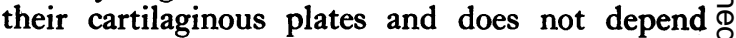
primarily upon changes in the disc itself. One feels that there is every justification for regarding ${ }^{\infty}$ cervical spondylosis as an affection both of the $\vec{\circ}$ bones and the intervertebral discs of the cervical spine. Once the degeneration of the disc has $\stackrel{\sigma}{\sigma}$ taken place, however, protrusion of one or other $\bar{C}$ portions of the disc with subsequent osteophyte 3 . formation may lead to the neural picture of pain in the arm provided the site of compression is, as has been previously indicated, in the region of primarily of the sensory nerve root.

It would be a convenient and very tidy situation if these three separate causative agents were in independent compartments. It becomes clear, however, as one studies cases of affection of the cervical spine, that all three factors may, in fact, be operative in any given case, and it is extremely $\vec{f}$ common for two at least to overlap. One always impressed by the frequency with which of. patient presenting with the symptoms of cervical root compression gives a previous history of a fall from a moving vehicle or even from a horse. The patient usually recalls that this occurred several years previously, and that he or she fell with the head in the flexed position. In these cases, one is struck by the fact that there is usually a single disc affection of the type described by Brain, Northfield and Wilkinson. ${ }^{6}$ One feels that in these circumstances the initial protrusion was itself asymptomatic, but the stage was set for the subsequent chain of processes of degeneration, osteophyte formation and subsequent calcification. It is appropriate to mention at this point Symonds's ${ }^{7}$ account of the role of trauma, or even ordinary movements, in precipitating frank neurological disabilities in patients who have cervical spondylosis-in other words, just as an injury may lead to a single disc type of spondylosis so the presence of the generalized osteoarthritic change or of degenerative changes associated with ageing may, in fact, allow mild trauma to produce very disabling neurological syndromes including that of the painful arm.

\section{Mechanism of Production of Symptoms}

Both Frykholm ${ }^{4}$ and Brain ${ }^{2}$ have stressed the development of ' root sleeve fibrosis' as a result of 
the changes enumerated above. This is said to consist of thickening of the dural root-sleeve with consequent obliteration of the root pouch and the production of a constricting ring of fibrous tissue round each nerve root. The constriction of the nerve roots and of the radicular nerve, with its associated ischaemia, leads on to degeneration of the nerve fibres. It seems very probable that root sleeve fibrosis may take place and produce a chronic arm pain, corresponding the formerly described entities of brachial neuritis and brachial neuralgia. On the other hand, it is difficult to escape the notion that direct compression of the nerve roots within the foramina may play some part in the production of radicular symptoms, and certainly in cases of acute onset following trauma this direct compression seems to play the more important role. The success of techniques of immobilization, either by surgical means or by the application of a collar, suggests that the actual mechanical affection of the nerve root by the abnormal bony tissue plays a not inconsiderable part in the production of symptoms and also runs counter to the notion that root sleeve fibrosis is the only factor at work:

\section{The Clinical Picture Produced by Cervical Spondylosis}

As indicated earlier, it is not proposed to set out all the syndromes which may result from this disorder and attention will be confined to the varying pictures of pain in the arm which may be associated with these bony and cartilaginous changes. The most common picture is that of a subacute onset of pain in the neck radiating into the arm and shoulder and very often assuming radicular or multiradicular distribution. At times, as was mentioned earlier, the onset may be rather more abrupt as a result of trauma. Clinical examination will often reveal reflex changes at one or more levels; frank sensory manifestations are unusual but the presence of lower motor neurone affection and even fibrillation in association with the pain is quite common. The following case history serves to illustrate this particular point.

\section{Case History}

A man, age sixty-two, complained of pain in the right thumb and forearm, particularly on attempting to use a screwdriver. After two weeks he began to experience difficulty even in winding his watch. The condition progressed and by the time he was seen three months after the onset of his symptoms, weakness of the hand was the predominant symptom though the pain had not improved. Clinical examination on 2.12.55 revealed that abnormalities were confined to the right hand and forearm and comprised diminution in the muscle bulk of the right thenar eminence, weakness of the opponens and abductor pollicis, and some loss of bulk with sparse fasciculation in the extensor group of the wrist and fingers. During the course of 1956 the condition progressed slightly and the loss of muscle bulk became more evident. $\mathrm{X}$-ray of the cervical spine on II.I.57 revealed evidence of disc degeneration at $\mathrm{C} / 5 / 6 / 7$ levels with associated anterior-osteophyte formation.

At this stage a plastic collar was applied. Within three months all his pain had disappeared and the power in the hand was almost normal. On 20.12.58 he was seen once more. His only complaint was of occasional pain in the right thumb. There was no evidence of any motor defect.

It is common for the patients to assert that their pain is made worse by cold weather, that it is invariably aggravated by activity and particularly by the performance of common household duties involving scrubbing or rubbing. In certain cases the pain may be referred much more widely than would have been anticipated by the radiological evidence of a single disc degeneration. Radiation to the upper cervical regions and even on to the chest wall is by no means unknown.

In certain cases the frozen-shoulder syndrome, with its gross limitation of active and passive movements of the shoulder, may be associated with cervical spondylosis, and usually develops after some days or weeks of pain due to the spondylosis itself. It is important to realize that this frozen-shoulder syndrome may require independent treatment including the peri-articular injection of appropriate steroids.

Brain, amongst others, has drawn attention to the fact that acro-paraesthesiae may occur as a symptom of cervical spondylosis. We have recently (Liversedge and Lees, in preparation) reviewed I 0 cases of acro-paraesthesiae and have been struck by the infrequency with which we have encountered the typical acro-paraesthetic picture with its characteristic nocturnal incidence in cases of cervical spondylosis. It is a point of additional interest that, although cervical spondylosis is fairly equally distributed between the two sexes, acro-paraesthesiae are exceedingly rare in men. It is our feeling that although radiological evidence of cervical spondylosis may be present in cases of acro-paraesthesiae it is likely that the two factors are merely co-incidental and that, although the spondylosis may contribute a little to the symptomatology, it is not the prime cause of the characteristic syndrome. Moreover, in certain cases the acro-paraesthesiae were dramatically relieved by carpal tunnel decompression despite the co-existence of obvious spondylotic changes on the radiographs. 


\section{Management of Pain in the Arm due to Cervical Spondylosis}

Because of the initial indecision about the prime cause of symptoms, various different types of treatment have been advised and tried but, on the whole, opinion - at least for the time being - is tending towards the non-operative conservative methods. We feel that manipulative techniques are positively harmful and should never be employed in the management of this type of disorder.

The first step is to establish the nature of the cervical abnormality, bearing in mind the three factors mentioned above. Cisternal myelography may be necessary, but this is used mainly as a means of excluding the existence of other forms of neural affection and in particular tumours of the roots or cord for which operation would be obligatory. Whatever the type of spondylotic process, the initial therapeutic action should consist of a period of immobilization in a suitable collar. Plaster, leather, plastic or other materials have been used. A felt-covered cardboard frame is the most comfortable type of collar and is best tolerated by most patients. One is impressed by the relative rapidity with which radicular symptoms settle in the majority of cases even with the simplest form of fixation. The collar should be so designed as to give the neck a slight degree of flexion. The patient is advised to wear it day and night for the first four to six weeks (although symptoms usually clear in the first seven to ten days). After that the collar may be left off for increasing periods during the next four weeks.

In a minority of cases this form of treatment may not be successful, and in this event it may be necessary to resort to a period of bed rest (usually Io to 14 days) with skull traction, followed by the application of one of the more rigid types of collar. Very occasionally the continuation of pain or the development of frank evidence of affection of the cord in the young 'single-disc' spondylotic may call for operation, but one feels that such action should be preceded by adequate trial of the conservative measures.

\section{REFERENCES}

I. BRAIN, W. R. (1948), Proc. roy. Soc. Med., 4I, 509.

2. BRAIN, W. R. (1954), Lancet, i, 687.

3. PAYNE, E. E., and SPILLANE, J. D. (1957), Brain, 89, 57 r.

4. FRYKHOLM, R. (I95 I), Acta chir. scand., ror, 345.

5. CAVE, A. J. E., GRIFFITHS, J. D., and WHITELEY, M. M. (1955), Lancet, i, 176.

6. BRAIN, W. R., NORTHFIELD, D. C., and WILKINSON, M. (1952), Brain, 75, 177.

7. SYMONDS, C. P. (1953), Lancet, i, 451 .

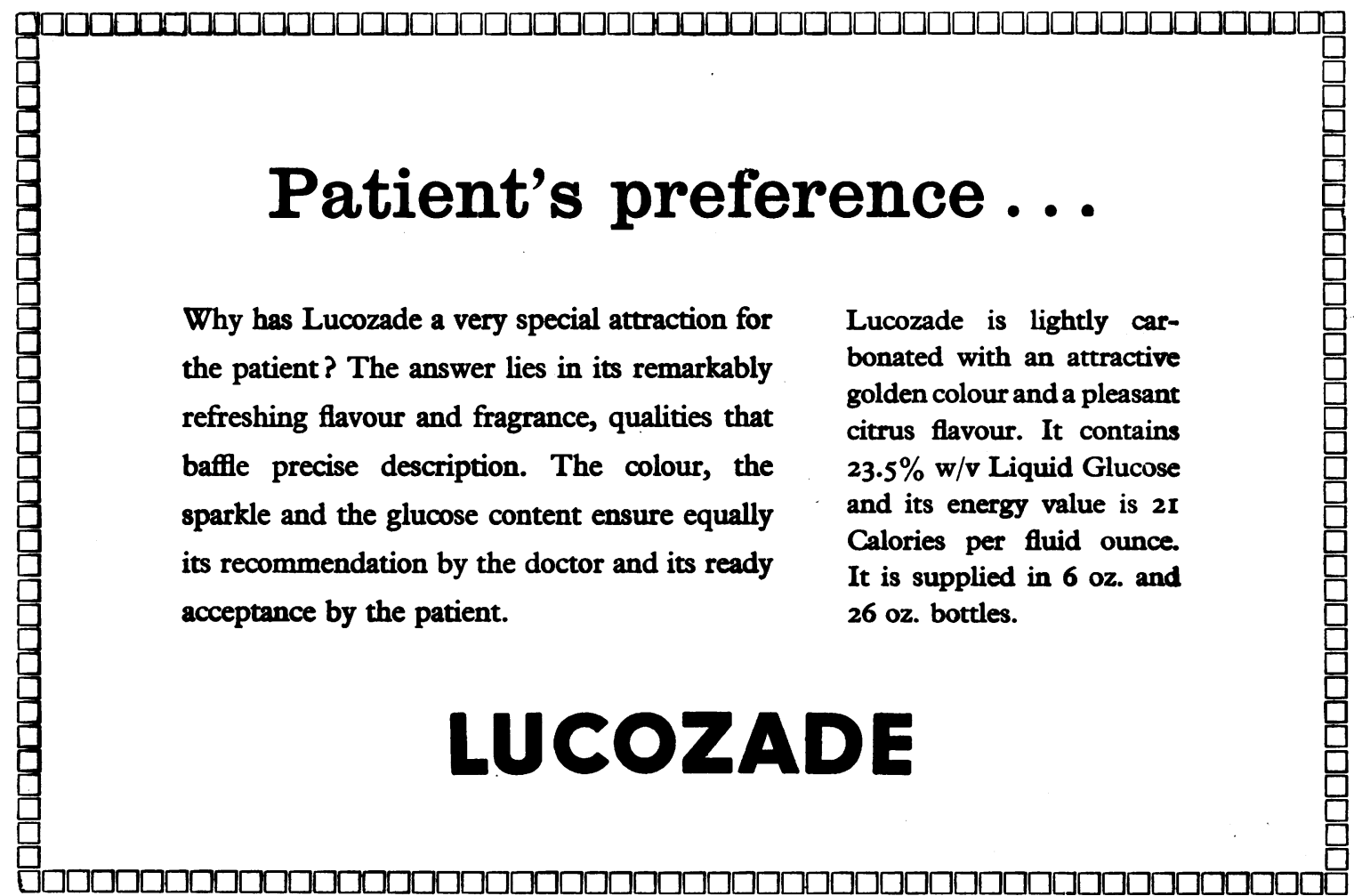

